\title{
THE SPECTRAL FUNCTION OF AN ELLIPTIC OPERATOR
}

\author{
BY \\ LARS HÖRMANDER \\ University of Lund, Lund, Sweden(1)
}

\section{Introduction}

In this paper we shall obtain the best possible estimates for the remainder term in the asymptotic formula for the spectral function of an arbitrary elliptic (pseudo-)differential operator. This is achieved by means of a complete description of the singularities of the Fourier transform of the spectral function for low frequencies.

In order to describe the results and methods more precisely we must recall some standard notations and hypotheses. Let $\Omega$ be a paracompact $C^{\infty}$ manifold and let $P$ ke an elliptic differential operator in $\Omega$ with $C^{\infty}$ coefficients. We assume that $P$ is formally positive, that is,

where $c>0$ and

$$
\begin{gathered}
(P u, u) \geqslant c(u, u), \quad u \in C_{0}^{\infty}(\Omega), \\
(u, v)=\int u \bar{v} d x
\end{gathered}
$$

for some positive $C^{\infty}$ density $d x$, kept fixed throughout. In the space $L^{2}(\Omega)$ obtained by completing $C_{0}^{\infty}(\Omega)$ in the norm $\|u\|=(u, u)^{\frac{1}{2}}$, the operator $P$ with domain $C_{0}^{\infty}(\Omega)$ is symmetric, and by a classical theorem of Friedrichs it has at least one self-adjoint extension $\hat{P}$ with a positive lower bound $c$. Let $\left\{E_{\lambda}\right\}$ be the spectral resolution of such an extension, and let $e(x, y, \lambda)$ be the kernel of $E_{\lambda}$. This is an element of $C^{\infty}(\Omega \times \Omega)$ called the spectral function of the self-adjoint extension $\hat{P}$.

Let $p$ be the principal symbol of $P$, which is a real homogeneous polynomial of degree $m$ on the cotangent bundle $T^{*}(\Omega)$. The measure $d x$ defines a Lebesgue measure $d \xi$ in each fiber of $T^{*}(\Omega)$; which is a vector space of dimension $n$. With the notation

$$
R(x, \lambda)=\lambda^{-n / m} e(x, x, \lambda)-(2 \pi)^{-n} \int_{B_{x}} d \xi
$$

where $B_{x}=\left\{\xi \in T_{x}^{*} ; p(\xi)<1\right\}$, our main result is

(1) This paper was written while the author was a member of the Institute for Advanced Study, Princeton, N. J. 
THEOREM 1.1. On every compact subset of $\Omega$ we have $R(x, \lambda)=O\left(\lambda^{-1 / m}\right)$ uniformly when $\lambda \rightarrow \infty$.

Many special cases of this theorem are known before. That $R(x, \lambda) \rightarrow 0$ when $\lambda \rightarrow \infty$ is a classical result of Carleman [3] in the second order case and Gårding [4] in general. When $P$ is an operator with constant coefficients in an open set in $\mathbf{R}^{n}$, the theorem is due to Gårding [5]. In the second order case it has been proved by Avakumovič [2] and in part by Lewitan $[10,11]$. Avakumovic also noted that for the Laplace operator on a sphere the jumps of $R(x, \lambda)$ caused by the high multiplicities of the eigenvalues are so large that no better error estimate is possible. (See Section 6 below.) Apart from these cases the best previous results are due to Agmon and Kannai [1] and Hörmander [8] who proved that $R(x, \lambda)=O\left(\lambda^{-\sigma / m}\right)$ for every $\sigma<1$ when the leading coefficients of $P$ are constant and for every $\sigma<\frac{1}{2}$ in the general case.

All of this work has been based on the study of the kernel of some function of $\hat{P}$ which satisfies a differential equation; information about the spectral function is then obtained by application of a Tauberian theorem. The following transforms have been used:

(i) The Stieltjes transform

$$
G_{1}(z)=\int(\lambda-z)^{-1} d E_{\lambda}
$$

which is defined when $z$ is not in the spectrum was considered already by Carleman [3]. We have $(\hat{P}-z) G_{1}(z)=1$, so $G_{1}$ is the resolvent of $\hat{P}$. When $z$ is outside an angle $|\arg z|<\varepsilon$, one can determine an asymptotic expansion of $G_{1}$ where the error terms are as small as any powers of $1 / z$, but this gives only the conclusion that $R(x, \lambda)=O(1 / \log \lambda)$. To obtain stronger results one must either determine $G_{1}$ outside such an angle with an exponentially small error or else one must produce good estimates much closer to the spectrum. (See Avakumovič [2], Agmon and Kannai [1], Hörmander [8].) This is not surprising since $d E_{\lambda}$ is essentially the jump of $G_{1}$ across the positive real axis.

(ii) The Laplace transform

$$
G_{2}(t)=\int e^{-t \lambda} d E_{\lambda}, t>0
$$

We have $(\partial / \partial t+\hat{P}) G_{2}(t)=0$ and $G_{2}(0)=1$, so $G_{2}$ can be regarded as a fundamental solution for the diffusion equation $(\partial / \partial t+\hat{P})$. This is the method used for example by Minakshisundaram and Pleijel [12] and Gårding [5]. If $t$ is real the results are parallel to those obtained by studying the resolvent outside an angle.

(iii) The $\zeta$-function

$$
G_{3}(x, y, s)=\int \lambda^{-s} d e(x, y, \lambda)
$$


The integral defines a continuous function when $\operatorname{Re} s>n / m$, and this function can be continued to a meromorphic function in the whole plane (Carleman [3], Minakshisundaram and Pleijel [12], Seeley [14]). If $G_{3}$ is represented in terms of $G_{1}$ or $G_{2}$ this follows from the properties of these transforms which are needed to prove that $R(x, \lambda) \rightarrow 0$. More precise error estimates are related to growth conditions on $G_{3}$ at infinity. However, these seem hard to prove directly, and as far as the author knows the study of $G_{3}$ has only been used to prove that $R(x, \lambda) \rightarrow 0$.

(iv) The work of Lewitan $[10,11]$ (and a long series of other papers) on the second order case is based on the study of the cosine transform

$$
G_{1}(t)=\int \cos t \sqrt{\lambda} d E_{\lambda}
$$

We have $\left(\partial^{2} / \partial t^{2}+\hat{P}\right) G_{4}(t)=0$ and $G_{4}(0)=1, G_{4}^{\prime}(0)=0$, so $G_{4}$ is closely related to the fundamental solution of the hyperbolic operator $\partial^{2} / \partial t^{2}+P$. (Note that $\partial^{2} / \partial t^{2}$ is a negative operator while $P$ is a positive operator, which makes the operator hyperbolic.) Avakumovič [2] also used his estimates of $G_{1}$ to draw conclusions concerning the Fourier transform

$$
G_{5}(t)=\int e^{-i t \lambda^{1 / m}} d E_{\lambda}
$$

when $m=2$. It was further proved in Hörmander [8] that the singularities of $G_{5}$ are local objects in the sense that the restrictions of the coefficients of $P$ to a neighborhood of a compact set $K \subset \Omega$ already determine the kernel of $G_{5}(t)$ on $K \times K$ for small $t$ modulo a $C^{\infty}$ function which is analytic with respect to $t$. This paper is based on the study of $G_{5}$.

The reason why the methods of Lewitan have not been applied to operators of order $m>2$ seems to be that the differential equation

$$
\left(i^{m} \partial^{m} / \partial t^{m}-\hat{P}\right) G_{5}(t)=0
$$

is not hyperbolic then. However, one can avoid this obstacle by considering the equation

$$
\left(i \partial / \partial t-\hat{P}^{1 / m}\right) G_{5}(t)=0
$$

which is obtained if irrelevant factors destroying the hyperbolicity are omitted. Here $\hat{P}^{1 / m}$ is defined by the spectral theorem. It is no longer a differential operator but is is a (classical) pseudo-differential operator (Seeley [14]) even if $P$ is only a pseudo-differential operator. This follows from the estimates used in methods (i) or (ii) to prove only that $R(x, \lambda) \rightarrow 0$. For the sake of simplicity we assume that $\Omega$ is compact, but in view of Theorem 5.3 in Hörmander [8] this is no essential restriction in the proof of Theorem 1.1. 
There are two reasons why the Fourier transform $G_{5}$ is particularly pleasant to work with. The first is that the Tauberian arguments needed to pass from information concerning the kernel of $G_{5}$ to the spectral function are extremely simple since there is an inversion formula for the Fourier transformation. The second and main reason is that the study of $G_{5}$ turns out to be very close to the proof of the generalized Huyghens principle for hyperbolic equations given by Lax [9]. Thus the singularities of the kernel of $G_{5}$ propagate with a finite speed. (Note that this would not have been the case if we had defined $G_{5}$ without taking an $m$ th root.) We recall that the main point in the arguments of Lax is the construction of a good approximation to the fundamental solution by means of the asymptotic expansions of geometrical optics. It is interesting to note that these methods were introduced to replace a classical construction of Hadamard for the second order case which was based on the use of normal coordinates. It is the latter technique which is the main tool in the work of Avakumovič [2], though he applied it to $G_{1}$ instead of $G_{5}$. Our constructions could also be used to study the resolvent $G_{1}$ rather than the Fourier transform $G_{5}$ but for the reasons indicated above the proofs would then be somewhat less simple and natural.

The plan of the paper is as follows. In Section 2 we discuss some of the main properties of operators of the type which occurs in the Lax construction of a parametrix for a hyperbolic operator. Further developments should yield an extension of the calculus of pseudo-differential operators with many applications to the study of non-elliptic differential operators. However, we have decided to discuss in this paper only the facts which we must use and a few results which seem to clarify them. Section 3 gives the construction of a parametrix of $i^{-1} \partial / \partial t+A$ where $A$ is a first order elliptic pseudo-differential operator on a compact manifold. Asymptotic properties of the spectral function of $A$ are then derived in Section 4, and the results are applied to differential operators in Section 5. In Section 6 finally we give an example of Avakumovič which shows that Theorem 1.1 cannot be improved in general.

\section{Fourier integral operators}

In this section we shall discuss some classes of operators containing both pseudodifferential operators (of type 1, 0 in the terminology of Hörmander [7]) and parametrices of hyperbolic operators. Our discussion will be local but everything we do is invariant so an extension to manifolds would not involve any difficulties.

As a motivation we first recall that a pseudo-differential operator in an open set $\Omega \subset \mathbf{R}^{n}$ is essentially defined by a Fourier integral operator 


$$
p(x, D) u(x)=(2 \pi)^{-n} \int p(x, \xi) e^{i\langle x, \xi\rangle} \hat{u}(\xi) d \xi, \quad u \in C_{0}^{\infty}(\Omega), x \in \Omega
$$

Here the symbol $p$ shall satisfy some conditions to be specified later. If we insert the definition of the Fourier transform $\hat{u}$, we obtain

$$
p(x, D) u(x)=(2 \pi)^{-n} \iint p(x, \xi) e^{i\langle x-y . \xi\rangle} u(y) d \xi d y
$$

The integral may not be absolutely convergent so it should be interpreted as a repeated integral: integration with respect to $y$ followed by integration with respect to $\xi$. Our purpose is to study operators defined by using a more general function in the exponent, but first we recall the usual condition on the symbol $p$.

Definition 2.1. If $\Omega$ is an open subset of $\mathbf{R}^{n}$ and $N$ is a positive integer, we denote by $S^{m}\left(\Omega, \mathbf{R}^{N}\right)$ the set of all $p \in C^{\infty}\left(\Omega \times \mathbf{R}^{N}\right)$ such that for every compact set $K \subset \Omega$ we have

$$
\left|D_{x}^{\beta} D_{\xi}^{\alpha} p(x, \xi)\right| \leqslant C_{K, \alpha, \beta}(1+|\xi|)^{m-|\alpha|}, \quad x \in K, \xi \in \mathbf{R}^{N} \text {. }
$$

The elements of $S^{m}$ are called symbols of order $m$. A subset $M$ of $S^{m}$ is said to be bounded if the same constants can be used in (2.2) for all $p \in M$.

On bounded subsets of $S^{m}$ the topology of pointwise convergence coincides with the topology of $C^{\infty}\left(\Omega \times \mathbf{R}^{N}\right)$ and will be referred to as the (weak) topology in what follows.

LEMMA 2.2. Every bounded set $M \subset S^{m}$ is contained in a bounded set $M^{\prime} \subset S^{m}$ where symbols vanishing for large $|\xi|$ are dense for the weak topology.

Proof. Let $\chi \in C_{0}^{\infty}\left(\mathbf{R}^{n}\right)$ be equal to 1 in a neighborhood of 0 . Then the set $M^{\prime}$ of all functions $p(x, \xi) \chi(\varepsilon \xi)$ with $p \in M$ and $0 \leqslant \varepsilon \leqslant 1$ has the required properties, for $|\xi||\alpha| D_{\xi}^{\alpha} \chi(\varepsilon \xi)$ is a bounded function of $\xi$ and $\varepsilon$ for every $\alpha$.

Remark. The space $S^{m}$ is the space of symbols of type 1,0 and order $m$ in the terminology used in Hörmander [7], and (2.1) is a pseudo-differential operator of order $m$ (and type 1,0$)$ if $p \in S^{m}$. It is often useful to note that $(2.2)$ means precisely that $\lambda^{-m} p(x, \lambda \xi)$ belongs to a bounded set in $C^{\infty}\left(\Omega \times\left\{\xi ; \frac{1}{2} \leqslant|\xi| \leqslant 2\right\}\right)$ when $\lambda \geqslant 1$. Occasionally we shall use the phrase $p \in S^{m}$ for large $|\xi|$ to mean that $p$ is defined at least when $|\xi|$ is large and that $(2.2)$ is valid for large $\xi$; this means that $\lambda^{-m} p(x, \lambda \xi)$ belongs to a bounded set in $C^{\infty}(\Omega \times$ $\left.\left\{\xi ; \frac{1}{2}<|\xi|<2\right\}\right)$ when $\lambda$ is large.

We shall now describe the conditions which should be satisfied by the function which is to replace the exponent $\langle x-y, \xi\rangle$ in $(2.1)^{\prime}$. 
Definition 2.3. Let $\Omega_{j}$ be an open set in $\mathbf{R}^{n_{j}}, j=1,2$. A real valued function $\varphi \in S^{1}\left(\Omega_{1} \times \Omega_{2}, \mathbf{R}^{N}\right)$ will be called a phase function if for every compact set $K \subset \Omega_{1} \times \Omega_{2}$ there is a constant $C$ such that

$$
\begin{array}{ll}
|\xi|^{2} \leqslant C\left(\sum_{1}^{n_{1}}\left|\partial \varphi / \partial x_{j}\right|^{2}+|\xi|^{2} \sum_{1}^{N}\left|\partial \varphi / \partial \xi_{j}\right|^{2}\right) ; & |\xi|>C,(x, y) \in K ; \\
|\xi|^{2} \leqslant C\left(\sum_{1}^{n_{2}}\left|\partial \varphi / \partial y_{j}\right|^{2}+|\xi|^{2} \sum_{1}^{N}\left|\partial \varphi / \partial \xi_{j}\right|^{2}\right) ; & |\xi|>C,(x, y) \in K .
\end{array}
$$

The purpose of these conditions is of course to guarantee that $e^{i \varphi(x, y, \xi)}$ is highly oscillatory for large $\xi$ even if $x$ or $y$ is fixed. In the case where $\varphi$ is a homogeneous function of $\xi$ of degree 1 for $|\xi| \geqslant 1$, which is really the only one that is essential for us, the condition (2.3) (or (2.4)) means that the restriction of $\varphi$ to the sphere bundle $\{(x, y, \xi) ;|\xi|=1\}$ has no zero which is a critical point if $x$ or $y$ is fixed.

Let $\varphi \in S^{1}\left(\Omega_{1} \times \Omega_{2}, \mathbf{R}^{N}\right)$ be a phase function. For every $p \in S^{m}\left(\Omega_{1} \times \Omega_{2}, \mathbf{R}^{N}\right)$ we wish to define a linear operator by

$$
P u(x)=\iint p(x, y, \xi) e^{i q(x, y, \xi)} u(y) d \xi d y, \quad u \in C_{0}^{\infty}\left(\Omega_{2}\right), \quad x \in \Omega_{1} .
$$

It is obvious that the double integral exists if $m<-N$. If $k$ is a non-negative integer such that $m+k<-N$ it is also obvious that $P$ is an integral operator with kernel $\epsilon C^{k}\left(\Omega_{1} \times \Omega_{2}\right)$. In particular, $P$ defines a continuous mapping $C_{0}\left(\Omega_{2}\right) \rightarrow C^{k}\left(\Omega_{1}\right)$. More generally, we have

THEOREM 2.4. For any $m$ there is a unique bilinear map

$$
S^{m}\left(\Omega_{1} \times \Omega_{2}, \mathbf{R}^{N}\right) \times \mathcal{E}^{\prime}\left(\Omega_{2}\right) \ni(p, u) \rightarrow P u \in \mathcal{D}^{\prime}\left(\Omega_{1}\right)
$$

such that $P u$ is given by (2.5) when $u$ is a function and the double integral is absolutely convergent, and in addition

(i) for any integers $\nu, \mu \geqslant 0$ with $m+N+\mu<\nu$ we obtain by restriction a bilinear mapping $S^{m} \times C_{0}^{\nu}\left(\Omega_{2}\right) \rightarrow C^{\mu}\left(\Omega_{1}\right)$ which is continuous when $p$ is restricted to a bounded subset of $S^{m}$ with the weak topology.

(ii) the map $S^{m} \times \mathcal{E}^{\prime \mu}\left(\Omega_{2}\right) \rightarrow \mathcal{D}^{\prime \prime}\left(\Omega_{1}\right)$ has the analogous continuity property.

Proof. The uniqueness is obvious in view of Lemma 2.2. If $\chi$ is the function used in its proof we even conclude that

$$
P u=\lim _{\varepsilon \rightarrow 0} \iint p(x, y, \xi) \chi(\varepsilon \xi) e^{i \varphi(x, y, \xi)} u(y) d \xi d y ;
$$

with the obvious interpretation of the integral this remains true for distributions $u$. 
To prove the existence it suffices to prove (i), for (ii) is just a dual statement. We have already seen that (i) is valid when $\nu=0$, so we can prove (i) assuming that $\nu>0$ and that the statement is proved for smaller values of $\nu$. First note that if $p$ vanishes for large $|\xi|$ then

$$
\begin{aligned}
& i \iint p \partial \varphi / \partial y_{j} e^{i \varphi} u d y d \xi=-\iint \partial p / \partial y_{j} e^{i \varphi} u d y d \xi-\iint p e^{i \varphi} \partial u / \partial y, d y d \xi \\
& i \iint p \partial \varphi / \partial \xi_{j} e^{i \varphi} u d y d \xi=-\iint \partial p / \partial \xi_{j} e^{i \varphi} u d y d \xi
\end{aligned}
$$

Next it follows from (2.4) that

$$
\psi=\left(\sum\left(\partial \varphi / \partial y_{j}\right)^{2}+\sum|\xi|^{2}\left(\partial \varphi / \partial \xi_{j}\right)^{2}\right)^{-1} \in S^{-2}
$$

(We define $\psi$ smoothly when $|\xi|<C$.) For large $|\xi|$ we have

$$
p=\sum q_{j} \partial \varphi / \partial y_{j}+\sum r_{j} \partial \varphi / \partial \xi_{j}
$$

where

$$
q_{j}=p \psi \partial \varphi / \partial y_{j} \in S^{m-1} \quad \text { and } \quad r_{j}=p \psi|\xi|^{2} \partial \varphi / \partial \xi, \in S^{m} \text {. }
$$

In view of (2.6) and (2.7) we can therefore rewrite $P u$ as a sum of operators of the type (2.5) of order $m-1$ acting on $u$ and $\partial u / \partial y_{j}, j=1, \ldots, n_{\mathbf{2}}$. Since $m-1+\mu<v-1$, this completes the induction proof of (i).

For later reference we also note the following consequence of (2.7):

Lтмм 2.5. The symbols $i \partial p / \partial \xi_{j}$ and $p \partial \varphi / \partial \xi_{j}$ define the same operator according to (2.5)'.

We shall next consider the singularities of the kernel $K_{P}$ of $P$. Let $\Omega_{\varphi}$ be the set of all $(x, y) \in \Omega_{1} \times \Omega_{2}$ such that for some constant $C$, depending on $(x, y)$,

$$
1 \leqslant C \sum_{\mathbf{1}}^{N}\left|\partial \varphi / \partial \xi_{j}\right|^{2}, \quad|\xi|>C
$$

(In the homogeneous case this means that 0 is not a critical value of the restriction of $\varphi$ to the unit sphere for fixed $x$ and $y$.) The set $\Omega_{\varphi}$ is always open. In fact, if $\omega$ is a compact neighborhood of $(x, y)$ the fact that $\varphi \in S^{1}$ implies that for $\left(x^{\prime}, y^{\prime}\right) \in \omega$

$$
\left|\partial \varphi\left(x^{\prime}, y^{\prime}, \xi\right) / \partial \xi_{j}-\partial \varphi(x, y, \xi) / \partial \xi_{j}\right| \leqslant C\left(\left|x-x^{\prime}\right|+\left|y-y^{\prime}\right|\right)
$$

for large $|\xi|$. If $(2.8)$ is valid at $(x, y)$ it follows with another constant for all $\left(x^{\prime}, y^{\prime}\right) \in \omega$ in a neighborhood of $(x, y)$. Let $F_{\varphi}$ be the complement of $\Omega_{\varphi}$ which is therefore closed.

THEOREM 2.6. The singular support of the distribution kernel $K_{P} \in \mathcal{D}^{\prime}\left(\Omega_{1} \times \Omega_{2}\right)$ of the operator defined by $(2.5)^{\prime}$ is always contained in $F_{\varphi}$.

13-682904 Acta mathematica. 121. Imprimé le 4 décembre 1968. 
Proof. We have to show that the kernel is a $C^{\infty}$ function in $\Omega_{\varphi}$. The proof is similar to that of Theorem 2.4. If $(x, y) \in \Omega_{\varphi}$, we have in a neighborhood of $(x, y)$ that

$$
\psi=\left(\sum\left|\partial \varphi / \partial \xi_{j}\right|^{2}\right)^{-1} \in S^{0}
$$

Since for large $|\xi|$

$$
p=p \psi \sum\left(\partial \varphi / \partial \xi_{j}\right)^{2}
$$

it follows from Lemma 2.5 that the kernel of $P$ near $(x, y)$ coincides with the kernel of an operator defined by a symbol of order $m-1$. If we repeat this argument we may conclude that $K_{P}$ coincides near $(x, y)$ with the kernel of an operator of arbitrary low order. In view of Theorem 2.4, this proves the theorem.

COROLLARY 2.7. If $u \in \mathcal{E}^{\prime}\left(\Omega_{2}\right)$ and $P$ is defined by $(2.5)^{\prime}$, then

$$
\text { sing supp } P u \subset F_{q} \text { sing supp } u \text {. }
$$

Here we have written for subsets $K$ of $\Omega_{2}$

$$
F_{\varphi} K=\left\{x \in \Omega_{1} ;(x, y) \in F_{\varphi} \text { for some } y \in K\right\} .
$$

Proof. That sing supp $P u \subset F_{p} \operatorname{supp} u$ follows immediately from the fact that $K_{P} \in C^{\infty}\left(\Omega_{\varphi}\right)$. Now $u$ can be written as a sum $u=v+w$ where $v \in C_{0}^{\infty}\left(\Omega_{2}\right)$ and supp $w$ is arbitrarily close to sing supp $u$. Since $P v \in C^{\infty}\left(\Omega_{1}\right)$ by Theorem 2.4, this proves the corollary.

The preceding result is of course the analogue of the pseudo-local property of pseudodifferential operators; in that case $\Omega_{1}=\Omega_{2}$ and $\varphi=\langle x-y, \xi\rangle$ so $F_{\varphi}$ is the diagonal.

Since we shall now start to vary $\varphi$ it is useful to have a notation for the class of operators of the form $(2.5)^{\prime}$.

Definition 2.8. If $\varphi$ is a phase function we denote by $L^{m}(\varphi)$ the class of operators $C_{0}^{\infty}\left(\Omega_{2}\right) \rightarrow C^{\infty}\left(\Omega_{1}\right)$ which modulo an operator with $C^{\infty}$ kernel can be written in the form (2.5)' for some $p \in S^{m}\left(\Omega_{1} \times \Omega_{2}, \mathbf{R}^{N}\right)$.

It is clear that $L^{m}(\varphi)$ only depends on the residue class of $\varphi$ modulo $S^{0}$. For assume that $q \in S^{0}$. Then $e^{i q} \in S^{0}$ and the operator defined by $p$ and the phase function $\varphi$ is equal to the operator defined by $p e^{-i q}$ and the phase function $\varphi+q$. Since $p e^{-i q}$ has the same order as $p$, this proves the assertion.

We shall now prove a less trivial result containing the fact that the class of pseudodifferential operators is invariant under diffeomorphisms. Let $I_{\varphi}$ be the ideal in $S^{0}$ generated by the derivatives $\partial \varphi / \partial \xi_{j}$, and let $J_{\varphi}$ be the functions of $(x, y)$ (locally) in the ideal $I_{\varphi}$. (We regard $I_{\varphi}$ as consisting of germs at $\xi=\infty$ so equality is only required for large $\left.|\xi|.\right)$ It is clear that $J_{\varphi}$ contains $C_{0}^{\infty}\left(\Omega_{\varphi}\right)$, and more precisely that $F_{\varphi}$ is the set of common zeros of the 
elements of $J_{\varphi}$. Let $J_{p}^{2} S^{1}$ denote the $S^{0}$ submodule of $S^{1}$ spanned by products of two elements of $J_{\varphi}$ and one of $S^{1}$.

THEOREM 2.9. If $\varphi_{1}$ and $\varphi$ are phase functions with $\varphi_{1}-\varphi \in J_{\varphi}^{2} S^{1}$, it follows that $L^{m}(\varphi) \subset$ $L^{m}\left(\varphi_{1}\right)$.

Note that there are no restrictions on $\varphi_{1}$ over $\Omega_{\varphi}$ so we cannot expect equality in general.

Before the proof we note a corollary:

CoRollary 2.10. Let $\Omega_{1}=\Omega_{2}$ and $N=\operatorname{dim} \Omega_{j}$; assume that $F_{\varphi}$ is the diagonal and that $\varphi(x, y, \xi)=\langle x-y, \xi\rangle+O\left(|x-y|^{2}|\xi|\right)$ at the diagonal. Then $L^{m}(\varphi)$ is equal to the space $L^{m}$ of pseudo-differential operators of order $m$ (and type 1,0).

To obtain Corollary 2.10 from Theorem 2.9 we first note that Taylor's formula applied to $\partial \varphi(y+t(x-y), y, \xi) / \partial \xi_{j}$ gives for $x$ close to $y$

$$
\partial \varphi(x, y, \xi) / \partial \xi_{j}=\left(x_{j}-y_{j}\right)+\sum a_{j k}(x, y, \xi)\left(x_{k}-y_{k}\right)
$$

where $a_{j k} \in S^{0}$ and $a_{j k}=0$ on the diagonal. It follows that $\operatorname{det}\left(\delta_{j k}+a_{j k}\right) \in S^{0}$ and equals I on the diagonal, which implies that $\left(\delta_{j k}+a_{j k}\right)$ has an inverse with matrix elements in $S^{0}$ in a neighborhood of the diagonal. Hence $\left(x_{j}-y_{j}\right) \in J_{\varphi}$, so if $\varphi_{1}$ is any other function satisfying the hypotheses of the corollary we have $L^{m}(\varphi) \subset L^{m}\left(\varphi_{1}\right)$. In view of the symmetry between $\varphi$ and $\varphi_{1}$ it follows that $L^{m}(\varphi)=L^{m}\left(\varphi_{1}\right)$ and taking $\varphi_{1}(x, y, \xi)=\langle x-y, \xi\rangle$ we obtain the corollary.

Proof of Theorem 2.9. Set $\varphi_{t}=\varphi+t\left(\varphi_{1}-\varphi\right), 0 \leqslant t \leqslant 1$, which causes no confusion for $t=1$. By hypothesis we can write

$$
\varphi_{1}-\varphi=\sum_{j, k=1}^{r} q_{j k} a_{j} a_{k}
$$

where $q_{j k} \in S^{1}$ and $a_{j} \in J_{\varphi}$ so that for some $b_{j p} \in S^{0}$

$$
a_{j}=\sum_{1}^{N} b_{j v} \partial \varphi / \partial \xi_{v}
$$

Our first purpose is to show that $\varphi$ can be replaced by $\varphi_{t}$ in (2.11). We have

$$
\sum b_{j p} \partial \varphi_{t} / \partial \xi_{\nu}=a_{j}+t \sum b_{j p} \partial\left(\varphi_{1}-\varphi\right) / \partial \xi_{\nu}=a_{j}+t \sum c_{j k} a_{k}
$$

where $c_{j k} \in S^{0}$ and $c_{j k}=0$ on $F_{\phi}$. Hence the matrix $\left(\delta_{j k}+t c_{j k}\right)$ has an inverse with elements in $S^{0}$ over a neighborhood of $F_{\varphi}$ for $0 \leqslant t \leqslant 1$. We need only consider operators in $L^{m}(\varphi)$ of 
the form (2.5)' where the support of $p$ belongs to that neighborhood, for the part of $p$ away from $F_{\varphi}$ only contributes an operator with $C^{\infty}$ kernel. Thus we have in the support of $p$

$$
a_{j}=\sum_{1}^{N} b_{j \nu}^{t} \partial \varphi_{t} / \partial \xi_{v}
$$

where $b_{j y}^{t} \in S^{0}$ and depends continuously on $t$ for $0 \leqslant t \leqslant 1$.

Now consider

$$
P_{t} u(x)=\iint p(x, y, \xi) e^{i \varphi_{t}(x, y, \xi)} u(y) d \xi d y
$$

If the support of $p$ is sufficiently close to $F_{\varphi}$, as we may assume, then $\varphi_{t}$ satisfies (2.3), (2.4) there uniformly with respect to $t \in[0,1]$. We have

$$
d^{r} P_{t} u / d t^{r}=\iint p(x, y, \xi) i^{r}\left(\varphi_{1}-\varphi\right)^{r} e^{i \varphi_{t}} u(y) d \xi d y
$$

Here we substitute for $\left(\varphi_{1}-\varphi\right)^{r}$ the sum given by (2.10) and obtain a number of terms each of which is of order $m+r$ but contains $2 r$ factors $a_{j}$. But if we replace one factor $a_{j}$ by the expression (2.12) it follows from Lemma 2.5 that one can reduce the order by one unit without affecting the other factors $a_{j}$. Repeating this argument $2 r$ times we conclude that $d^{r} P_{t} u / d t^{r}$ is actually in $L^{m-r}\left(\varphi_{t}\right)$, hence as smooth as we wish uniformly with respect to $t$ for large $r$.

$$
\text { If we write } \quad Q_{j}=(-1)^{j} d^{j} P_{t} / d t^{j} /\left.j !\right|_{t=1} \in L^{m-j}\left(\varphi_{1}\right),
$$

it follows from Taylor's formula that

$$
P_{0}=\sum_{0}^{k-1} Q_{j}+(-1)^{k} \int_{0}^{1} t^{k-1} /(k-1) ! d^{k} P_{t} / d t^{k} d t
$$

Let $Q$ be an operator in $L^{m}\left(\varphi_{1}\right)$ defined by an asymptotic sum of the symbols defining $Q_{j}, j=0,1,2, \ldots$ (see Theorem 2.7 in Hörmander [7]). Then $P_{0}-Q$ has an infinitely differentiable kernel, which proves that $P_{0} \in L^{m}\left(\varphi_{1}\right)$. The proof is complete. Clearly the proof could also give formulas for the symbol of $Q$ but we have no need for them here.

Remark. If $\varphi, \varphi_{1}$ and $p$ are asymptotically sums of homogeneous functions of $\xi$, then $Q$ is also defined by means of such a kernel.

Corollary 2.10 is of course closely related to the invariance of the class of pseudodifferential operators under a change of variables. (See also Hörmander [6], [7].)

We shall now pass to the study of multiplicative properties of $L(\varphi)$. The main point is the following lemma. 
Lемм 2.11. Let $q(x, D)$ be a compactly supported pseudo-differential operator of order $\mu$ (and type 1,0 ) in $\Omega$. Let $\Psi$ be a compact subset of $C^{\infty}(\Omega, \mathbf{R})$ such that no element of $\Psi$ has a critical point, and let $\mp$ be a compact set in $C^{\infty}(\Omega)$. For $\psi \in \Psi$ we set

$$
\psi(z)=\psi(x)+\langle z-x, \operatorname{grad} \psi(x)\rangle+\varrho_{x}(z) .
$$

Then we have for every positive integer $N$

$$
e^{-i \lambda \psi} q(x, D)\left(f e^{i \lambda \psi}\right)=\sum_{|\alpha|<N} q^{(\alpha)}(x, \lambda \operatorname{grad} \psi(x)) D_{z}^{\alpha}\left(f(z) e^{i \lambda Q_{x}(z)}\right) /\left.\alpha !\right|_{z=x}+\lambda^{\mu-N / 2} R_{N}(x, \lambda),
$$

where $R_{N}$ lies in a bounded set in $C(\Omega)$ for $\psi \in \Psi, f \in \mathcal{F}, \lambda \geqslant 1$.

Proof. This is actually what was verified in the proof of Theorem 2.16 of Hörmander [7], although that theorem as stated does not contain Lemma 2.11. (Naturally one should also be able to obtain the lemma from Corollary 2.10 above.) For homogeneous symbols Lemma 2.11 is Theorem 3.3 in [6].

The following consequence of Lemma 2.11 will allow us to apply a pseudo-differential 'p srator inder the sign of integration in (2.5).

THEOREM 2.12. Let $q(x, D)$ be a compactly supported pseudo-differential operator of order $\mu$ (and type 1,0$)$ in $\Omega_{1}$. Let $\varphi \in S^{1}\left(\Omega_{1} \times \Omega_{2}, \mathbf{R}^{N}\right)$ and assume that on compact subsets of $\Omega_{1} \times \Omega_{2}$

$$
|\xi|^{2} \leqslant C \sum\left|\partial \varphi / \partial x_{j}\right|^{2}, \quad|\xi|>C .
$$

$W e$ set $\varphi(z, y, \xi)=\varphi(x, y, \xi)+\left\langle z-x, \operatorname{grad}_{x} \varphi(x, y, \xi)\right\rangle+\varrho(x, y, z, \xi)$. For $p \in S^{m}\left(\Omega_{1} \times \Omega_{2}, \mathbf{R}^{N}\right) w e$ have then

$$
e^{-i \varphi} q(x, D)\left(p e^{i \varphi}\right)=\sum_{|\alpha|<N} q^{(\alpha)}\left(x, \operatorname{grad}_{x} \varphi\right) D_{z}^{\alpha}\left(p(z, y, \xi) e^{i \varphi}\right) /\left.\alpha !\right|_{z=x}+R_{N}(x, y, \xi)
$$

where $R_{N} \in S^{m+\mu-N / 2}\left(\Omega_{1} \times \Omega_{2}, \mathbf{R}^{N}\right)$.

Proof. We may restrict $y$ to a compact subset $K$ of $\Omega_{2}$ and assume that $|\xi| \geqslant 1$. Since the hypotheses of Lemma 2.11 are satisfied by the closures $\mathcal{I}$ and $\Psi$ of the set of functions

$$
f(x)=\lambda^{-m} p(x, y, \lambda \xi), \quad \psi(x)=\lambda^{-1} \varphi(x, y, \lambda \xi) ; \quad y \in K,|\xi|=1, \lambda \geqslant 1,
$$

we conclude that

$$
R_{N}(x, y, \lambda \xi) \lambda^{-m-u+N / 2}
$$

is bounded in $C\left(\Omega_{1}\right)$ when $y \in K$ and $|\xi|=I, \lambda \geqslant 1$. The statement now follows from Theorem 2.9 in Hörmander [7] if we note that the general term in the sum in (2.14) is in $S^{m+\mu-|\alpha| / 2}$, in fact in $S^{m+\mu-(|\alpha|+1) / 2}$ if $|\alpha|$ is odd. (See the beginning of the proof of Theorem 2.16 in [7].) 
Terms of order $\geqslant m+\mu-1$ can only occur in (2.14) when $|\alpha| \leqslant 2$, and for $|\alpha|=2$ they only occur when both $z$-derivatives fall on $e^{i e}$. This gives

COROLLARY 2.13. Let the hypotheses be as in Theorem 2.12 and set

$$
b=\sum_{|\alpha|=2} q^{(\alpha)}\left(x, \operatorname{grad}_{x} \varphi\right) D_{x}^{\alpha} i \varphi / \alpha ! \in S^{\mu-1} .
$$

Then we have

$$
e^{-i \varphi} q(x, D)\left(p e^{i \varphi}\right)=\sum_{|\alpha|<2} q^{(\alpha)}\left(x, \operatorname{grad}_{x} \varphi\right) D_{x}^{\alpha} p+b p+R(x, y, \xi)
$$

where $R \in S^{m+\mu-2}$.

If $\varphi$ satisfies (2.13) it is clear that we can form the composition of $q(x, D)$ and the operator (2.5) by operating under the integral sign, and by Theorem 2.12 the product will again be in the same class. In fact, operation under the integral sign is legitimate when $p$ vanishes for large $\xi$ and is therefore justified in general by continuity arguments based on Lemma 2.2. However, the condition (2.13) is not a natural one since it would not be invariantly defined if we replace $\Omega_{1}$ and $\Omega_{2}$ by manifolds and $\Omega_{1} \times \Omega_{2} \times \mathbf{R}^{N}$ by a real vector bundle over the product. On the other hand the condition (2.3) is invariant and it is the only one that is really needed. For the contributions from the part where $|\partial \varphi / \partial \xi|$ is bounded from below give an operator with a $C^{\infty}$ kernel so it is clear that one can split $P$ in a sum where one term has a $C^{\infty}$ kernel and the other only involves integration over a set where (2.13) is valid. This proves that the product of a compactly supported pseudo-differential operator of order $\mu$ in $\Omega_{1}$ and an operator in $L^{m}(\varphi)$ is always in $L^{m+\mu}(\varphi)$. Analogously we have multiplication to the right by pseudo-differential operators in $\Omega_{2}$, which follows by taking adjoints. The details are left for the reader to supply.

\section{The Fourier transform of the spectral measure}

Let $\Omega$ be a compact $C^{\infty}$ manifold with a given positive $C^{\infty}$ density $d x$, and let $A$ be a formally self-adjoint semibounded elliptic pseudo-differential operator of order 1 (and type 1,0 ) in $\Omega$. In local coordinate systems $A$ is thus (modulo operators with $C^{\infty}$ kernel) of the form $a(x, D)$ where $a \in S^{1}$, the limit

$$
a^{0}(x, \xi)=\lim _{t \rightarrow \infty} t^{-1} a(x, t \xi)
$$

exists when $\xi \neq 0$, and

$$
a^{0}(x, \xi)>0, \xi \neq 0 \text {. }
$$

(Since $a \in S^{1}$ the limit (3.1) exists in the $C^{\infty}$ topology on the set where $\xi \neq 0$.) If the given density $d x$ agrees with the Lebesgue measure in the local coordinates, the formula for the 
adjoint of a pseudo-differential operator (Theorem 2.15 in [7]) shows that $\operatorname{Im} a \in S^{0}$. The closure in $L^{2}(\Omega)$ of $A$ with domain $C^{\infty}(\Omega)$ is a self-adjoint semibounded operator. We shall denote it by $\mathcal{A}$; the domain is equal to $H_{(1)}(\Omega)$. (For the definition of the space $H_{(s)}$ see e.g. Hörmander [7], section 2.6.) The notation $A$ will also be used for the extension of the pseudo-differential operator $A$ to $\mathcal{D}^{\prime}(\Omega)$.

Let $\left\{d E_{\lambda}\right\}$ be the spectral resolution of $\mathcal{A}$ and form the Fourier transform

$$
\hat{E}(t)=\int e^{-i t \lambda} d E_{\lambda}=e^{-i t A}
$$

$\hat{E}(t)$ is thus a one-parameter group of unitary operators. Moreover, $\hat{E}(t)$ defines a continuous mapping of $H_{\{s\rangle}(\Omega)$ into itself for any integer $s$, and this mapping is strongly continuous as a function of $t$. This follows from the fact that if $A \geqslant c$, then $\hat{E}$ commutes with $(A-c+1)^{s}$ for any $s$ and the $H_{(s)}$ norm of $u$ is equivalent to the $L^{2}$ norm of $(A-c+1)^{s} u$. Note that for $u_{0} \in H_{(1)}$, the unique solution of the equation

$$
D_{t} u+\mathcal{A} u=0
$$

with initial data $u(0)=u_{0}$ is given by $u(t)=\hat{E}(t) u_{0}$.

Our purpose is to determine the singularities of the kernel $\hat{E}(x, t, y)$ for small $|t|$. To do so we shall use the techniques of section 2 to construct a parametrix for the operator $D_{t}+A$, which solves the initial value problem for this equation approximately. Let $\omega_{1}$ be a local coordinate patch in $\Omega$ which we identify with the corresponding open set in $\mathbf{R}^{n}$. We assume that the given density in $\Omega$ agrees with the Lebesgue measure in $\omega_{1}$ and choose $a \in S^{1}\left(\omega_{1}, \mathbf{R}^{n}\right)$ so that $a(x, D)$ is compactly supported in $\omega_{1}$ and $A-a(x, D)$ has a $C^{\infty}$ kernel in $\omega_{1}$. (Cf. Hörmander [7], pp. 148-149.) Let $\omega$ be a relatively compact open subset of $\omega_{1}$. We want to find an approximate representation of the operator $\hat{E}(t)$ of the form

$$
(Q(t) f)(x)=\iint q(x, t, y, \xi) e^{i \varphi(x, t, y, \xi)} f(y) d y d \xi, \quad f \in C_{0}^{\infty}(\omega)
$$

where $q$ shall vanish for $x$ outside some compact subset of $\omega_{1}$ so that $Q(t) f$ can be extended to be 0 in $\Omega \backslash \omega_{1}$ without introduction of any singularities.

The construction will rely on Corollary 2.13 - and is of course essentially the same as that in Lax [9]. The first step is to choose the phase function $\varphi$ as in geometrical optics, that is, so that

$$
\partial \varphi / \partial t+a\left(x, \operatorname{grad}_{x} \varphi\right) \in S^{0}
$$

which makes the term in (2.16) with $\alpha=0$ harmless. Furthermore, we want $Q(0)$ to be the identity operator, and in order to be able to check this using Corollary 2.10 we require that 


$$
\varphi(x, 0, y, \xi)=\langle x-y, \xi\rangle+O\left(|x-y|^{2}|\xi|\right) \quad \text { when } x \rightarrow y \text {. }
$$

However, we shall now differ from Lax [9] by demanding that $\varphi$ shall be linear with respect to $t$, which is natural in view of the translation invariance of the operator $D_{t}+A$ with respect to $t$. Thus we set with some function $a^{\prime}$ to be chosen later

$$
\varphi(x, t, y, \xi)=\psi(x, y, \xi)-t a^{\prime}(y, \xi)
$$

The condition (3.6) then becomes

$$
\psi(x, y, \xi)=\langle x-y, \xi\rangle+O\left(|x-y|^{2}|\xi|\right) \quad \text { when } x \rightarrow y,
$$

and (3.5) gives when $x=y$

$$
a^{\prime}(y, \xi)-a(y, \xi) \in S^{0}
$$

From (3.7) it follows that we may replace (3.5) by

$$
a^{\prime}\left(x, \operatorname{grad}_{x} \psi\right)-a^{\prime}(y, \xi) \in S^{0} .
$$

Definition 3.1. A phase function $\psi \in S^{1}\left(U, \mathbf{R}^{n}\right)$ where $U$ is a neighborhood of the diagonal in $\bar{\omega} \times \bar{\omega}$ and a real valued function $a^{\prime} \in S^{1}\left(\omega_{1}, \mathbf{R}^{n}\right)$ will be called adapted to $A$ if $(3.5)^{\prime},(3.6)^{\prime}$ and (3.7) are fulfilled in $U$ and $\omega_{1}$ respectively.

It is obvious that a real valued function $a^{\prime}$ satisfying (3.7) exists if and only if $\operatorname{Im} a \in S^{0}$ -one can then take $a^{\prime}=\operatorname{Re} a$-and we have seen above that this is true if $A$ is formally self-adjoint. The condition $(3.5)^{\prime}$ is independent of the choice of $a^{\prime}$. Clearly $(3.5)^{\prime}$ and $(3.6)^{\prime}$ are implied by

$$
a^{\prime}\left(x, \operatorname{grad}_{x} \psi\right)=a^{\prime}(y, \xi),
$$

$$
\psi(x, y, \xi)=0 \text { when }\langle x-y, \xi\rangle=0 \text { and } \operatorname{grad}_{x} \psi(x, y, \xi)=\xi \text { when } x=y \text {. }
$$

These equations define a Cauchy problem for the non-linear first order differential equation $a^{\prime}\left(x, \operatorname{grad}_{x} \psi\right)=a^{\prime}(y, \xi)$, depending on the parameters $y$ and $\xi$. If $a^{\prime}$ were homogeneous with respect to $\xi$ we could restrict $\xi$ to the compact set $|\xi|=1$, and then extend the definition of $\psi$ by homogeneity. In the general case we set $\xi=\lambda \eta$ where $\frac{1}{2} \leqslant|\eta| \leqslant 2$ and $\lambda$ is large. With $\psi=\lambda \chi$, the conditions (3.5)", $(3.6)^{\prime \prime}$ become

$$
\begin{gathered}
\lambda^{-1} a^{\prime}\left(x, \lambda \operatorname{grad}_{x} \chi\right)=\lambda^{-1} a^{\prime}(y, \lambda \eta), \\
\chi=0 \text { when }\langle x-y, \eta\rangle=0, \operatorname{grad}_{x} \chi=\eta \text { when } x=y .
\end{gathered}
$$

Since $\lambda^{-1} a^{\prime}(x, \lambda \vartheta) \rightarrow a^{0}(x, \vartheta)$ in the $C^{\infty}$ topology for $\vartheta \neq 0$ when $\lambda \rightarrow \infty$, this is also defined for $\lambda=\infty$ and we now have a compact parameter space. From the standard existence theorems for first order partial differential equations it follows that for large $\lambda$ there exists a unique solution in a fixed neighborhood of the diagonal, independent of $\lambda$ and $\eta$, and the solution 
belongs to a bounded set of $C^{\infty}$ functions of $x, y$ and $\eta$ for large $\lambda$. In a neighborhood $U$ of the diagonal of $\bar{\omega} \times \bar{\omega}$ in $\omega_{1} \times \omega_{1}$ we have therefore constructed a function $\psi \in S^{1}$ such that $\psi$ and $a^{\prime}$ are adapted to $A$. (See a remark following Lemma 2.2.)

From (3.6) and the hypothesis that $\psi \in S^{1}$ it follows if $U$ is replaced by a smaller neighborhood of the diagonal of $\bar{\omega} \times \bar{\omega}$ that

$$
\begin{gathered}
|\xi| \leqslant C\left|\operatorname{grad}_{x} \psi\right| ; \quad|\xi| \geqslant C,(x, y) \in U ; \\
|x-y| \leqslant C\left|\operatorname{grad}_{\xi} \psi\right| ; \quad|\xi| \geqslant C,(x, y) \in U .
\end{gathered}
$$

In what follows we assume that $U$ is chosen so that these estimates are valid. This was tacitly assumed already in the passage from (3.5) to (3.5)'.

Let $\chi \in C_{0}^{\infty}(U)$ be a function which is equal to 1 in an open neighborhood $U_{0}$ of the diagonal in $\bar{\omega} \times \bar{\omega}$. We shall choose the function $q$ in (3.4) so that $q=0$ except over a compact subset of $U$, so it will not matter that $\psi$ is not defined everywhere. Since the set $F_{\varphi}$ in Theorem 2.6 is a closed subset of $U \times \mathbf{R}$ and is contained in the diagonal when $t=0$, it follows that we can choose $\varepsilon>0$ so that $(x, y) \in U_{0}$ if $(x, y, t) \in F_{\varphi},|t|<\varepsilon$ and $y \in \bar{\omega}$. Thus we need not be concerned with the definition of $q$ outside $U_{0}$, fot it can only contribute an operator with a $C^{\infty}$ kernel when $|t|<\varepsilon$.

In view of Corollary 2.10 and the proof of Theorem 2.9 we can choose a kernel $I \in S^{0}\left(U, \mathbf{R}^{n}\right)$ vanishing outside a compact subset of $U$ such that $I-(2 \pi)^{-n} \in S^{-1}\left(U_{0}, \mathbf{R}^{n}\right)$ and

$$
f \rightarrow \iint I(x, y, \xi) e^{i \varphi(x, y, \xi)} f(y) d y d \xi-f(x), \quad f \in C_{0}^{\infty}(\omega), x \in \Omega
$$

is an integral operator with $C^{\infty}$ kernel. In order that the operator (3.4) for $t=0$ shall represent the identity operator (modulo operators with $C^{\infty}$ kernel) we therefore pose the boundary condition

$$
q(x, 0, y, \xi)=I(x, y, \xi)
$$

Furthermore, in order that $\left(D_{t}+a(x, D)\right) Q(t)$ shall have a $C^{\infty}$ kernel we must make sure that

$$
e^{-i \varphi}\left(D_{t}+a(x, D)\right)\left(q e^{i \varphi}\right) \in S^{-\infty}\left(U_{0}, \mathbf{R}^{n}\right)
$$

To solve (3.10), (3.11) we shall use Corollary 2.13. With $b$ defined by (2.15) set $b_{1}=b+a\left(x, \operatorname{grad}_{x} \psi\right)-a^{\prime}(y, \xi) \in S^{0}\left(U, \mathbf{R}^{n}\right)$. We shall successively determine kernels $q_{0}, q_{1}, \ldots$ in $\omega_{1} \times(-\varepsilon, \varepsilon) \times \omega \times \mathbf{R}^{n}$ so that for large $|\xi|$

$$
\begin{gathered}
\sum_{|\alpha|=1} a^{(\alpha)}\left(x, \operatorname{grad}_{x} \psi\right) D_{x}^{\alpha} q_{0}+D_{t} q_{0}+b_{1} q_{0}=0 \\
q_{0}(x, 0, y, \xi)=I(x, y, \xi)
\end{gathered}
$$




$$
\begin{gathered}
e^{-i \varphi}\left(D_{t}+a(x, D)\right)\left(q_{k} e^{i \varphi}\right)-\sum_{|\alpha|=1} a^{(\alpha)}\left(x, \operatorname{grad}_{x} \psi\right) D_{x}^{\alpha} q_{k}-D_{t} q_{k}-b_{1} q_{k}+R_{k}(x, t, y, \xi)=0, \\
\sum_{|\alpha|=1} a^{(\alpha)}\left(x, \operatorname{grad}_{x} \psi\right) D_{x}^{\alpha} q_{k}+D_{t} q_{k}+b_{1} q_{k}=\chi R_{k-1}(x, t, y, \xi), k \geqslant 1 \\
q_{k}(x, 0, y, \xi)=0, k \geqslant 1
\end{gathered}
$$

Thus we argue in each step as if there were no remainder term in (2.16) and $a\left(x, \operatorname{grad}_{x} \psi\right)=$ $a^{\prime}(y, \xi)$, but we take care of the error in the following step. Since $a^{(\alpha)}\left(x, \operatorname{grad}_{x} \psi\right)$ is real valued and has a uniform bound for $|\alpha|=1$, it is clear that for sufficiently small $\varepsilon$ these recursion formulas have solutions with compact support in $U$ for $|t|<\varepsilon$; as in the discussion of the function $\psi$ above we conclude first that $q_{0} \in S^{0}$, hence that $R_{0} \in S^{-1}$ (Corollary 2.13), then that $q_{1} \in S^{-1}$; inductively we obtain $q_{j} \in S^{-i}, R_{j} \in S^{-j-1}$. Adding (3.12), (3.14), (3.15) gives

$$
e^{-i \varphi}\left(D_{t}+a(x, D)\right)\left(\left(q_{0}+\ldots+q_{k}\right) e^{i \varphi}\right)=\chi \sum_{0}^{k-1} R_{j}-\sum_{0}^{k} R_{j}=(\chi-1) \sum_{0}^{k-1} R_{j}-R_{k},
$$

and adding (3.13), (3.16) we find that

$$
\left(q_{0}+\ldots+q_{k}\right)(x, 0, y, \xi)=I(x, y, \xi) .
$$

Now define $q \sim \sum_{0}^{\infty} q_{j}$ (see Hörmander [7], Theorem 2.7). It follows immediately that $q$ has the required properties (3.10), (3.11). Thus $Q$ maps $C_{0}^{\infty}(\omega)$ into $C^{\infty}(\Omega \times(-\varepsilon, \varepsilon))$;

$$
Q(0) f=f+k f, \quad f \in C_{0}^{\infty}(\omega)
$$

where $k$ has a $C^{\infty}$ kernel, and for $|t|<\varepsilon$ we have

$$
\left(D_{t}+A\right) Q(t) f=K(t) f, \quad f \in C_{0}^{\infty}(\omega),
$$

where $K$ has a $C^{\infty}$ kernel as a function of $t$ also. To prove (3.18) we note that $A$ differs from $a(x, D)$ by an operator with $C^{\infty}$ kernel in $\omega_{1}$, so $K(t)$ has a $C^{\infty}$ kernel over $\omega_{1} \times \omega$. Since $A$ is pseudo-local the kernel is also $C^{\infty}$ over $\Omega \times \omega$ outside a compact subset of $U$, hence over $\Omega \times \omega$.

From (3.17) and (3.18) we conclude that for $|t|<\varepsilon$

or more briefly

$$
\begin{gathered}
Q(t) f=\hat{E}(t)(f+k f)+\int_{0}^{t} \hat{E}(s) K(t-s) f d s, \\
Q(t)-\hat{E}(t)=\hat{E}(t) k+\int_{0}^{t} \hat{E}(s) K(t-s) d s,
\end{gathered}
$$

as an equality between operators from $C_{0}^{\infty}(\omega)$ to $C^{\infty}(\Omega)$. Since $\hat{E}(t)$ is a $C^{\infty}$ function of $t$ with values in the space of continuous mappings of $C^{\infty}(\Omega)$ into itself, we conclude that $Q(t)-\hat{E}(t)$ has a $C^{\infty}$ kernel in $\Omega \times(-\varepsilon, \varepsilon) \times \omega$. 


\section{Asymptotic properties of the spectral function}

Having determined the singularities of the kernel of the Fourier transform $\widehat{E}(t)$ of the spectral measure when $|t|<\varepsilon$, we shall now invert the Fourier transformation to obtain precise information concerning the spectral function at infinity. Let $\varrho$ be a positive function in $S(\mathbf{R})$ such that supp $\hat{\varrho} \subset(-\varepsilon, \varepsilon)$ and $\hat{\varrho}(0)=1$. If $\hat{E}(x, t, y)$ and $Q(x, t, y)$ denote the distribution kernels of $\hat{E}(t)$ and of the operator $Q(t)$ of the form (3.5) constructed in the preceding section, we have found that

$$
\hat{\varrho}(t)(\widehat{E}(x, t, y)-Q(x, t, y)) \in C^{\infty}, \quad x \in \Omega, y \in \omega
$$

and the support of this function is bounded in the $t$-direction. Hence

$$
\int \varrho(\lambda-\mu) d e(x, y, \mu)-\mathcal{F}^{-1}(\varrho(.) Q(x, ., y))(\lambda)
$$

is a rapidly decreasing function when $\lambda \rightarrow \infty$, uniformly with respect to $x \in \Omega$ and to $y$ in a compact subset of $\omega$. Here we have assumed that the local coordinates are chosen so that the given density in $\Omega$ is the Lebesgue measure in $\omega$.

To evaluate the inverse Fourier transform in (4.1) we introduce

$$
R(x, \lambda, y, \xi)=(2 \pi)^{-1} \int \hat{\varrho}(t) q(x, t, y, \xi) e^{i t \lambda} d t
$$

This is a function in $S^{0}\left(\Omega \times \mathbf{R} \times \omega, \mathbf{R}^{n}\right)$ and it is rapidly decreasing as a function of $\lambda$ in the sense that $\lambda^{N} R(x, \lambda, y, \xi)$ and its translates with respect to $\lambda$ belong to a bounded set in $S^{0}$ for each integer $N$. This follows immediately if we multipy (4.2) by $\lambda^{N}$ and integrate by parts with respect to $t$.

If $q$ had compact support in $\xi$ we would have

$$
Q(x, t, y)=\int q(x, t, y, \xi) e^{i\left(y(x, y, \xi)-a^{\prime}(y, \xi) t\right)} d \xi,
$$

so the inverse Fourier transform would be

$$
\mathcal{F}^{-1}(\hat{\varrho}(.) Q(x, ., y))(\lambda)=\int R\left(x, \lambda-a^{\prime}(y, \xi), y, \xi\right) e^{i \varphi(x, y . \xi)} d \xi
$$

In view of the rapid decrease of $R$ as a function of $\lambda$ this integral is a well defined continuous function of $x, y, \lambda$ for any $q \in S^{0}$, and using Lemma 2.2 as in Section 2 we conclude that (4.3) is valid in general. Thus we have proved: 
Lемм А 4.1. If $R$ is defined by (4.2) with the function $q$ constructed in Section 3 , then

$$
\int \varrho(\lambda-\mu) d e(x, y, \mu)-\int R\left(x, \lambda-a^{\prime}(y, \xi), y, \xi\right) e^{i \psi(x, y, \xi)} d \xi
$$

is rapidly decreasing as $\lambda \rightarrow \infty$.

To obtain the asymptotic formulas we must study the function $R$ more closely. With the notation

we have for any integer $N$

$$
m(y, \sigma)=m\left\{\xi ; a^{\prime}(y, \xi) \leqslant \sigma\right\}
$$

$$
\left|\int R\left(x, \lambda-a^{\prime}(y, \xi), y, \xi\right) e^{i \psi(x, y, \xi)} d \xi\right| \leqslant C \int(1+|\lambda-\sigma|)^{-N} d m(y, \sigma) .
$$

To estimate the integral on the right we need a simple lemma.

LEM M 4.2. The function $m(y, \lambda)$ belongs to $S^{n}(\omega, \mathbf{R})$ for large $\lambda$.

Proof. Set $\lambda=t \mu$ where $\frac{1}{2} \leqslant \mu \leqslant 2$ and $t$ is large. Then

$$
t^{-n} m(y, t \mu)=m\left\{\xi ; t^{-1} a^{\prime}(y, t \xi) \leqslant \mu\right\} .
$$

Since $t^{-1} a^{\prime}(y, t \xi) \rightarrow a^{0}(y, \xi)$ in the $C^{\infty}$ topology for $\xi \neq 0$ when $t \rightarrow \infty$, it follows from the implicit function theorem that the function defining the surface $t^{-1} a^{\prime}(y, t \xi)=\mu$ in polar coordinates also converges in the $C^{\infty}$ topology when $t \rightarrow \infty$. Hence

$$
t^{-n} m(y, t \mu) \rightarrow \mu^{n} m\left\{\xi ; a^{0}(y, \xi) \leqslant 1\right\}
$$

in the $C^{\infty}$ topology, which proves the statement.

By Lemma 4.2 we have $|d m / d \sigma| \leqslant C \sigma^{n-1}$ for large $\sigma$, hence if $N>n$

$$
\begin{aligned}
\int(1+|\lambda-\sigma|)^{-N} d m(y, \sigma) & =O\left(\lambda^{-N}\right)+C \int(1+|\lambda-\sigma|)^{-N}(1+|\sigma|)^{n-1} d \sigma \\
& =O\left(\lambda^{-N}\right)+C(1+|\lambda|)^{n-1} \int(1+|\lambda-\sigma|)^{n-1-N} d \sigma \\
& =O\left(\lambda^{-N}\right)+C^{\prime}(1+|\lambda|)^{n-1}
\end{aligned}
$$

Here we have used that $(1+|\sigma|) \leqslant(1+|\lambda|)(1+|\sigma-\lambda|)$ in view of the triangle inequality. When $x=y$ we can therefore conclude from (4.4) and the positivity of $\varrho$ and of de that

$$
e(x, x, \lambda+1)-e(x, x, \lambda) \leqslant C(1+|\lambda|)^{n-1},
$$

when $x$ is in a compact subset of $\omega$, hence for all $x \in \Omega$. Since $e(x, y, \lambda+1)-e(x, y, \lambda)$ is the kernel of a positive operator, this result can immediately be extended to points outside the diagonal (cf. Lemma 3.1 in Hörmander [8]) so we have proved the crucial 
LEMMA 4.3. There is a constant $C$ such that

$$
|e(x, y, \lambda+1)-e(x, y, \lambda)| \leqslant C(1+|\lambda|)^{n-1} .
$$

Next we shall estimate the spectral function by integrating (4.4) from $-\infty$ to $\lambda$. This gives

$$
\left|\int \varrho(\lambda-\mu) e(x, y, \mu) d \mu-\iint_{\sigma<\varrho} R\left(x, \sigma-a^{\prime}(y, \xi), y, \xi\right) e^{i \psi(x, y, \xi)} d \xi d \sigma\right| \leqslant C
$$

From (4.6) we obtain

$$
\left|\int \varrho(\lambda-\mu) e(x, y, \mu) d \mu-e(x, y, \lambda)\right| \leqslant C(1+|\lambda|)^{n-1}
$$

if we note that $(4.6)$ implies

$$
|e(x, y, \lambda+\mu)-e(x, y, \lambda)| \leqslant C(1+|\lambda|+|\mu|)^{n-1}(1+|\mu|) .
$$

In order to derive an estimate for $e(x, y, \lambda)$ it only remains to study the double integral in (4.7). To do so we note that by the definition of $R$

$$
\int R(x, \sigma, y, \xi) d \sigma=\hat{\varrho}(0) q(x, 0, y, \xi)=I(x, y, \xi)
$$

(The notation $I$ is explained in Section 3.) Set

$$
\begin{aligned}
& R_{1}(x, \tau, y, \xi)=\int_{-\infty}^{\tau} R(x, \sigma, y, \xi) d \sigma, \tau<0 \\
& R_{1}(x, \tau, y, \xi)=\int_{-\infty}^{\tau} R(x, \sigma, y, \xi) d \sigma-I(x, y, \xi)=\int_{\infty}^{\tau} R(x, \sigma, y, \xi) d \sigma, \tau>0 .
\end{aligned}
$$

Then $R_{1}(x, \lambda, y, \xi) \lambda^{N}$ is uniformly bounded for any $N$ and we have

$$
\begin{aligned}
\iint_{\sigma<\lambda} R\left(x, \sigma-a^{\prime}(y, \xi), y, \xi\right) e^{i \psi(x, y, \xi)} d \xi d \sigma \\
\quad=\int_{a^{\prime}(y, \xi)<\lambda} I(x, y, \xi) e^{i \psi(x, y, \xi)} d \xi+\int R_{1}\left(x, \lambda-a^{\prime}(y, \xi), y, \xi\right) e^{i \psi(x, y, \xi)} d \xi .
\end{aligned}
$$

By an estimate analogous to (4.5) and by Lemma 4.2 the last integral can be bounded by $C(1+|\lambda|)^{n-1}$. Since $I(x, y, \xi)-(2 \pi)^{-n}=O(1 /|\xi|)$ when $(x, y) \in U_{0}$ and $\xi \rightarrow \infty$, it follows when $(x, y) \in U_{0}$ that

$$
\left|\int_{a(y, \xi)<\lambda} I(x, y, \xi) e^{i \psi(x, y, \xi)} d \xi-(2 \pi)^{-n} \int_{a^{\prime}(y, \xi)<\lambda} e^{i \psi(x, y, \xi)} d \xi\right| \leqslant C(1+|\lambda|)^{n-1} .
$$

On the other hand, we have $I=0$ when $(x, y) \notin U$. Hence we have proved 
THEOREM 4.4. For $x$ and $y$ in a compact subset of the coordinate patch $\omega$ and sufficiently close we have uniformly

$$
\left|e(x, y, \lambda)-(2 \pi)^{-n} \int_{a^{\prime}(y, \xi)<\lambda} e^{i \psi(x, y, \xi)} d \xi\right| \leqslant C(1+|\lambda|)^{n-1}
$$

if the phase function $\psi$ and $a^{\prime}$ are adapated to $A$ over a neighborhood of the diagonal in $\omega \times \omega$ (Definition 3.1). In particular,

$$
\left|e(x, x, \lambda)-(2 \pi)^{-n} \int_{a^{\prime}(x, \xi)<\lambda} d \xi\right| \leqslant C(1+|\lambda|)^{n-1}
$$

uniformly in $\Omega$. On compact subsets of the complement of the diagonal in $\Omega \times \Omega$ we have also

$$
|e(x, y, \lambda)| \leqslant C(1+|\lambda|)^{n-1}
$$

Here the local coordinates are assumed to be chosen so that the Lebesgue measure in the local coordinates is equal to the given positive density in the manifold. In the proof of (4.11) we choose $U$ so that $(x, y) \notin U$.

Remark. In many cases it is not necessary to make a very careful choice of the phase function $\psi$ in (4.9). For example, if the surface $\left\{\xi ; a^{0}(x, \xi)=1\right\}$ has only positive curvatures and $n \geqslant 3$, it follows easily from (4.9) that in local coordinate systems

$$
\left|e(x, y, \lambda)-(2 \pi)^{-n} \int_{a^{\prime}(y, \xi)<\lambda} e^{i\langle x-y, \xi\rangle} d \xi\right| \leqslant C(1+|\lambda|)^{n-1} .
$$

In the one dimensional case on the other hand there is hardly any freedom at all in the choice of $\psi$.

We shall now study the Riesz means $e^{\alpha}(x, x, \lambda)$. (See Hörmander [8] for the definitions.) To do so we must evaluate the double integral in (4.7) more precisely. We now choose the function $\varrho \in S$ so that $\operatorname{supp} \underline{\varrho} \subset(-\varepsilon, \varepsilon)$ and $\hat{\varrho}=1$ in $(-\varepsilon / 2, \varepsilon / 2)$. (Clearly $\varrho$ cannot be positive then.) First note that

$$
S(x, \sigma, \lambda)=\int_{a^{\prime}(x, \xi)<\lambda} R(x, \sigma, x, \xi) d \xi
$$

belongs to $S^{n}(\omega \times \mathbf{R}, \mathbf{R})$ for large $\lambda$ and is rapidly decreasing as a function of $\sigma$. The proof is essentially a repetition of that of Lemma 4.2 : We set $\lambda=t \mu$ where $\frac{1}{2} \leqslant \mu \leqslant 2$ and $t$ is large and obtain

$$
t^{-n} S(x, \sigma, t \mu)=\int_{t^{-1} a^{\prime}(x, t \xi)<\mu} R(x, \sigma, x, t \xi) d \xi
$$


The only new feature is that the integral on the right could cause trouble for small $\xi$. This difficulty is eliminated if one subtracts a function $S_{1}$ defined as $S$ but with $a^{\prime}(x, \xi)$ replaced by a homogeneous function independent of $x$, for the assertion is easily proved for $S_{1}$. We leave the details for the reader.

Now we rewrite the double integral in (4.7) with $x=y$ as follows

$$
\iint_{\sigma<\lambda} R\left(x, \sigma-a^{\prime}(x, \xi), x, \xi\right) d \xi d \sigma=\iint_{\sigma<\lambda-a^{\prime}(x, \xi)} R(x, \sigma, x, \xi) d \xi d \sigma=\int S(x, \sigma, \lambda-\sigma) d \sigma .
$$

Since $S(x, \sigma, \lambda)$ is rapidly decreasing as a function of $\sigma$, the contribution to the integral when $|\sigma|>\lambda / 2$ is rapidly decreasing. For $|\sigma|<\lambda / 2$ we have by Taylor's formula for any $N$, since $S \in S^{n}$,

$$
S(x, \sigma, \lambda-\sigma)=\sum_{0}^{k-1}(-\sigma)^{j} / j ! d^{j} S(x, \sigma, \lambda) / d \lambda^{j}+O\left((1+|\sigma|)^{-N} \lambda^{n-k} \sigma^{k}\right) .
$$

The integral of the error terms is $O\left(\lambda^{n-k}\right)$, and

$$
\int_{|\sigma|<\lambda / 2}(-\sigma)^{j} d^{j} S(x, \sigma, \lambda) / d \lambda^{j} d \sigma=\int_{-\infty}^{\infty}(-\sigma)^{j} d^{j} S(x, \sigma, \lambda) / d \lambda^{j} d \sigma+O\left(\lambda^{-N}\right)
$$

for any $N$. The infinite integral we can express in terms of the Fourier transform in view of (4.2) and this gives the expression

$$
d^{j} /\left.d \lambda^{j} \int_{a^{\prime}(x, \xi)<\lambda} D_{t}^{j} q(x, t, x, \xi) d \xi\right|_{t=0}
$$

which is a function in $S^{n-j}(\omega, \mathbf{R})$. Hence the asymptotic sum

$$
e_{0}(x, \lambda) \sim \sum_{0}^{\infty}\left(-i \partial^{2} / \partial t \partial \lambda\right)^{j} /\left.j ! \int_{a^{\prime}(x, \xi)<\lambda} q(x, t, x, \xi) d \xi\right|_{t=0}
$$

is defined. We choose $e_{0}$ equal to 0 on the negative half axis and set as usual for $\operatorname{Re} \alpha \geqslant 0$

$$
e_{0}^{\alpha}(x, \lambda)=\int^{\lambda}(1-\mu / \lambda)^{\alpha} d e_{0}(x, \mu)
$$

If $\varphi(x, \lambda)$ is a continuous function on $\Omega \times \mathbf{R}$ which is rapidly decreasing when $\lambda \rightarrow \infty$, that is, $O\left(\lambda^{-N}\right)$ for every $N$, we set

$$
\varphi^{\alpha}(x, \lambda)=\int_{-\infty}^{\lambda}(1-\mu / \lambda)^{\alpha} \varphi(x, \mu) d \mu .
$$

Note that $\varphi^{\alpha}$ is bounded and has an asymptotic expansion in non-negative integral powers of $1 / \lambda$ at $+\infty$. 
THEOREM 4.5. If $e_{0}$ satisfies (4.12) there exists a continuous function $\varphi(x, \lambda)$ on $\Omega \times \mathbf{R}$ which is rapidly decreasing when $\lambda \rightarrow \infty$ such that for $\operatorname{Re} \alpha>0$

$$
\left|e^{\alpha}(x, x, \lambda)-e_{0}^{\alpha}(x, \lambda)-\varphi^{\alpha}(x, \lambda)\right| \leqslant C_{\alpha}(1+|\lambda|)^{n-1-\mathrm{Re} \alpha}
$$

We have $\int \varphi(x, \lambda) d \lambda=-k(x, x)$ if $k$ is the operator in (3.17).

The presence of the function $\varphi$ in (4.13) is required since $e_{0}$ only takes into account the symbol of the operator $A$. The effect of adding to $A$ an operator with a $C^{\infty}$ kernel is accounted for by the term $\varphi^{\alpha}$.

Proof of Theorem 4.5. The definition of $e_{0}$ and (4.1) mean that

$$
\varrho * d e(x, \lambda)-d e_{0}(x, \lambda)=\varphi(x, \lambda) d \lambda,
$$

where $\varphi$ is rapidly decreasing and $\int \varphi(x, \lambda) d \lambda=(\hat{E}(x, t, y)-Q(x, t, y))_{t=0, x=y}=-k(x, x)$. Hence $d e-d e_{0}-\varphi d \lambda=(\delta-\varrho) * d e$, or

$$
e-e_{0}-\varphi^{0}=(\delta-\varrho) * e=f .
$$

Now it follows from (4.8) that

$$
|f(\lambda)|=\mid(\delta-\varrho) * e(\lambda)) \mid \leqslant C(1+|\lambda|)^{n-1}
$$

and by (4.14) we also know that $f$ is rapidly decreasing at $-\infty$. Furthermore $f$ has no spectrum in the neighborhood of 0 where $\hat{\varrho}=1$. It follows that

$$
\left|f^{\alpha}(\lambda)\right| \leqslant C(1+|\lambda|)^{n-1-\operatorname{Re} \alpha}
$$

(See e.g. Hörmander [8], Theorem 2.6.) Since $e^{\alpha}-e_{0}^{\alpha--} \varphi^{\alpha}=f^{\alpha}$, the theorem is proved.

\section{The case of differential operators}

In Sections 3 and 4 we have studied the spectral function of an elliptic pseudo-differential operator of order 1 on a compact manifold. We shall now prove analogous statements for differential operators of order $m$. As in the introduction we shall consider a positive self-adjoint extension $\hat{P}$ of an elliptic differential operator with $C^{\infty}$ coefficients on a paracompact manifold $\Omega$ of dimension $n$. In a local coordinate patch $\omega$ where the Lebesgue measure agrees with the given density in $\Omega$ we choose a function $\psi(x, y, \xi)$ for $x$ and $y$ close to each other so that $\psi$ is homogeneous of degree 1 with respect to $\xi \in \mathbf{R}^{n}$ and

$$
p\left(x, \operatorname{grad}_{x} \psi\right)=p(y, \xi), \quad \psi(x, y, \xi)=\langle x-y, \xi\rangle+O\left(|x-y|^{2}|\xi|\right) \quad \text { as } x \rightarrow y .
$$

Here $p$ is the principal symbol of $P$. 
THEOR $\mathrm{E}$ 5.1. For $x$ and $y$ in a compact subset of $\omega$ and sufficiently close we have uniformly

$$
\left|e(x, y, \lambda)-(2 \pi)^{-n} \int_{D(y, \xi)<\lambda} e^{i \psi(x, y, \xi)} d \xi\right| \leqslant C(1+|\lambda|)^{(n-1) / m}
$$

if $\psi$ is homogeneous in $\xi$ of degree 1 and satisfies (5.1). In particular,

$$
\left|e(x, x, \lambda)-(2 \pi)^{-n} \int_{p(x, \xi)<\lambda} d \xi\right| \leqslant C(1+|\lambda|)^{(n-1) / m}
$$

uniformly in compact subsets of $\Omega$. On compact subsets of the complement of the diagonal in $\Omega \times \Omega$ we have also

$$
|e(x, y, \lambda)| \leqslant C(1+|\lambda|)^{(n-1) / m}
$$

Proof. Note first of all that by Theorem 5.3 in Hörmander [8] it suffices to prove the theorem when $\Omega$ is compact, for example a torus. Set $\mathcal{A}=\hat{P}^{1 / m}$, which is a pseudo-differential operator of order 1 whose symbol $a(x, \xi)$ is asymptotically the sum of $p(x, \xi)^{1 / m}$ and homogeneous functions of order $0,-1,-2, \ldots$ (Seeley [14]). Since $\psi$ and $p^{1 / m}$ are adapted to $\mathcal{A}$ the theorem now follows from Theorem 4.4. In particular, we have proved Theorem 1.1.

Theorem 5.1 allows us to apply Theorems 6.1-6.4 in Hörmander [8]. For example, by Theorems 6.1 and 6.2 there we obtain the following localization theorem.

THEOREM 5.2. If $f \in L^{p}(\Omega), 1 \leqslant p \leqslant 2$, and if $f$ has compact support in case $p<2$, it follows that the Riesz means $e^{\alpha}(x, f, \lambda)$ of the eigenfunction expansion of $f$ converge to 0 locally uniformly in the complement of the support of $f$ when $\operatorname{Re} \alpha \geqslant(n-1) / p$.

For further references and applications we refer the reader to [8]. However, we shall give some remarks here concerning the Riesz means $e^{\alpha}(x, x, \lambda)$ of the spectral function on the diagonal. First note that the function $e_{0}$ in Theorem 4.5 is asymptotically a sum of integral powers of $\lambda$ if the symbol $a$ of $\mathcal{A}$ is such a sum of homogeneous terms of integral order. Hence Theorem 4.5 applied to $\hat{P}^{1 / m}$ gives, again in view of Theorem 5.2 in Hörmander [8], that the Riesz means of $e\left(x, x, \lambda^{m}\right)$ can be approximated by a sum of integral powers of $\lambda$ within an error which is $O\left(\lambda^{n-1-\text { Re }}\right)$. Now

$$
\begin{aligned}
e^{\alpha}\left(x, x, \lambda^{m}\right) & =\int^{\lambda}\left(1-\tau^{m} / \lambda^{m}\right)^{\alpha} d e\left(x, x, \tau^{m}\right) \\
& =\int^{\lambda}\left[m(1-\tau / \lambda)-\left(\begin{array}{c}
m \\
2
\end{array}\right)(1-\tau / \lambda)^{2}+\ldots+(-1)^{m-1}(1-\tau / \lambda)^{m}\right]^{\alpha} d e\left(x, x, \tau^{m}\right)
\end{aligned}
$$

14-682904 Acta mathematica. 121. Imprimé le 4 décembre 1968. 
so results on the Riesz means of $e\left(x, x, \lambda^{m}\right)$ can be carried over to statements on the Riesz means $e^{\alpha}(x, x, \lambda)$. (See also the proof of Theorem 2.7 in [8].) Thus $e^{\alpha}(x, x, \lambda)$ differs from $a$ finite sum of integral powers of $\lambda^{1 / m}$ by an error which is $O\left(\lambda^{(n-1-\operatorname{Re} \alpha) / m}\right)$. To compute the coefficients it is easier to use the standard techniques of pseudo-differential operators; we refer the reader to Theorem 5.2 in [8] for such formulas. They can also be obtained from the asymptotic expansion of the fundamental solution of the heat equation $\partial / \partial t+P$ on the diagonal at $t=0$ since this is the Abel mean of $e(x, x, t)$. Note that in contrast with Theorem 4.5 the coefficients can be expressed completely in terms of the symbol of $P$.

Our methods can be applied with no essential modification in the case of systems for which the eigenvalues of $p(x, \xi)$ are distinct. In particular, (5.4) remains valid for such systems and we have

$$
\lambda^{-n / m} e(x, x, \lambda)-(2 \pi)^{-n} \int p(\xi)^{-n / m} \omega(\xi)=O\left(\lambda^{-1 / m}\right)
$$

Here $\omega(\xi)$ is the differential form

$$
\omega(\xi)=n^{-1}\left(\xi_{1} d \xi_{2} \wedge \ldots \wedge d \xi_{n}+\ldots+(-1)^{n} \xi_{n} d \xi_{1} \wedge \ldots \wedge d \xi_{n-1}\right)
$$

in $\mathbf{R}^{n}$, carried over to $T_{x}^{*}$ by a linear map preserving the Lebesgue measure. The integration takes place over the sphere $\left(T_{x}^{*}-\{0\}\right) / \mathbf{R}_{+}$, oriented by $\omega>\mathbf{0}$.

However for systems with multiple eigenvalues we have no information beyond the results of Agmon-Kannai [1] and Hörmander [8].

\section{A counterexample}

It was pointed out by Avakumovič [2] that it is not possible to improve Theorem 1.1 for the Laplacean on the sphere $S^{3} \subset \mathbf{R}^{4}$. For the sake of completeness we shall recall his arguments here for the case of the $n$-sphere. First we give some well-known facts concerning spherical harmonics. (See e.g. Müller [13].)

If in $\mathbf{R}^{n+1}$ we use the polar coordinates $x=r \omega$ where $r \in \mathbf{R}_{+}$and $\omega \in S^{n}$, the Laplace operator assumes the form

$$
\Delta=r^{-2} \Delta_{S}+\partial^{2} / \partial r^{2}+\frac{n}{r} \partial / \partial r
$$

outside the origin, where $\Delta_{S}$ is the Laplacean in $S^{n}$. If $u(x)=r^{\mu} v(\omega)$ is a homogeneous function of degree $\mu$, it follows that outside the origin

$$
\Delta u=r^{-2+\mu}\left(\Delta_{S} v+\mu(\mu+n-1) v\right)
$$

Hence $\Delta u=0$ outside the origin if and only if $v$ is an eigenfunction of $-\Delta_{S}$ with eigenvalue $\lambda=\mu(\mu+n-1)$. Since $\lambda$ describes all values $\geqslant 0$ when $\mu \leqslant 1-n$, we obtain all eigenfunctions 
of $-\Delta_{S}$ by restricting to $S^{n}$ all distributions $u$ in $\mathbf{R}^{n}$ which are harmonic and homogeneous of degree $\leqslant 1-n$ outside the origin. Then $\Delta u$ has to be a linear combination of the derivatives of the Dirac measure, so we conclude that $\mu=1-n-k$ where $k$ is an integer $\geqslant 0$ and that

$$
u(x)=\sum_{|\alpha|=k} a_{\alpha} D^{\alpha} E
$$

where $E$ is the fundamental solution of $\Delta$ and $a_{\alpha}$ are constants. (When $k=0, n=1$, the logarithmic potential $E$ must be replaced by a constant.) The Fourier transform of $u$ is $\sum a_{\alpha} \xi^{\alpha}|\xi|^{-2}$, so it follows that $u$ is supported by the origin if and only if $|\xi|^{2}$ divides the polynomial $\sum a_{\alpha} \xi^{\alpha}$. Let $N_{k}$ be the dimension of the space of homogeneous polynomials of degree $k$ in $n+1$ variables,

$$
N_{k}=\left(\begin{array}{c}
n+k \\
n
\end{array}\right)
$$

We define $N_{k}=0$ for $k<0$. Then it follows that the multiplicity of the eigenvalue $\lambda_{k}=k(k+n-1)$ of $-\Delta_{S}$ is $N_{k}-N_{k-2}$ for $k=0,1, \ldots$ If $V_{n}$ is the volume of $S^{n}$ and $e$ is the spectral function of $-\Delta_{S}$, it follows that

$$
\begin{gathered}
e\left(x, x, \lambda_{k}+0\right)-e\left(x, x, \lambda_{k}-0\right)=\left(N_{k}-N_{k-2}\right) / V_{n} \\
e\left(x, x, \lambda_{k}+0\right)=\left(N_{k}+N_{k-1}\right) / V_{n}
\end{gathered}
$$

Since $N_{k}-N_{k-2}$ is a polynomial in $k$ of degree $n-1$, it follows from (6.1) that

$$
e\left(x, x, \lambda_{k}+0\right)-e\left(x, x, \lambda_{k}-0\right) \geqslant c \lambda_{k}^{(n-1) / 2}
$$

for some $c>0$ and large $k$. This shows that Theorem 1.1 cannot be improved. From (6.2) we also obtain that

$$
e(x, x, \lambda) \lambda^{-n / 2}-2 /\left(n ! V_{n}\right)=O\left(\lambda^{-\frac{1}{2}}\right)
$$

which confirms Theorem 1.1 in this special case, if we note that

$$
2 /\left(n ! V_{n}\right)=(2 \pi)^{-n} V_{n-1} / n
$$

and that $V_{n-1} / n$ is the volume of the unit ball in $\mathbf{R}^{n}$.

\section{References}

[1]. Agmon, S. \& KannaI, Y., On the asymptotic behavior of spectral functions and resolvent kernels of elliptic operators. Israel J. Math., 5 (1967), 1-30.

[2]. Avakumovič, V. G., Über die Eigenfunktionen auf geschlossenen Riemannschen Mannigfaltigkeiten. Math. Z., 65 (1956), 327-344.

[3]. Carleman, T., Propriétés asymptotiques des fonctions fondamentales des membranes vibrantes. C. R. Sème Congr. des Math. Scand. Stockholm 1934; 34-44 (Lund 1935). 
[4]. GÅRDING, L., On the asymptotic distribution of the eigenvalues and eigenfunctions of elliptic differential operators. Math. Scand., 1 (1953), 237-255.

[5]. — On the asymptotic properties of the spectral function belonging to a self-adjoint semi-bounded extension of an elliptic differential operator. Kungl. Fysiogr. Sällsk. $i$ Lund Förh., 24, No. 21 (1954), 1-18.

[6]. Hörmander, L., Pseudo-differential operators. Comm. Pure Appl. Math., 18 (1965), 501-517.

[7]. — Pseudo-differential operators and hypoelliptic equations. Amer. Math. Soc. Proc. Symp. Pure Math., 10 (1968), 138-183.

[8]. - On the Riesz means of spectral functions and eigenfunction expansions for elliptic differential operators. Recent Advances in the Basic Sciences, Yeshiva University Conference November 1966, 155-202 (to appear).

[9]. LAX, P. D., Asymptotic solutions of oscillatory initial value problems. Duke Math. $J$., 24 (1957), 627-646.

[10]. Lewrtan, B. M., On the asymptotic behavior of the spectral function of a self-adjoint differential equation of the second order. Izv. Akad. Nauk SSSR Sér. Mat., 16 (1952), 325-352.

[11]. - On the asymptotic behavior of the spectral function and the eigenfunction expansion of self-adjoint differential equations of the second order II. Izv. Akad. Nauk SSSR Sér. Mat., 19 (1955), 33-58.

[12]. Minakshisundaram, S. \& Pleiset, $\AA$., Some properties of the eigenfunctions of the Laplace operator on Riemannian manifolds. Canad. J. Math. 4 (1952), 26-30.

[13]. MÜLLER, C., Spherical harmonics. Springer-Verlag lecture notes in mathematics, 17 (1966).

[14]. Seeley, R. T., Complex powers of an elliptic operator. Amer. Math. Soc. Proc. Symp. Pure Math., 10 (1968), 288-307.

Received May 7, 1968 